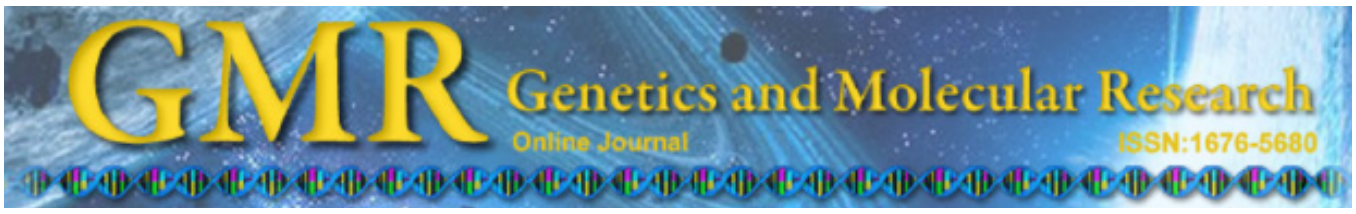

\title{
RAPD-based genetic diversities and correlation with morphological traits in Camellia (Theaceae) cultivars in China
}

\author{
X.F. Wang ${ }^{1}$, H.Y. Zheng ${ }^{1}$,W.H. Zheng ${ }^{1}$, C.Q. Ao ${ }^{1}$, H.Y. Jin ${ }^{1}$, L.H. Zhao ${ }^{1}$, \\ N. $\mathbf{L i}^{1}$ and L.R. Jia ${ }^{2}$ \\ ${ }^{1}$ College of Life and Environmental Sciences, Wenzhou University, \\ Wenzhou, Zhejiang, P.R. China \\ ${ }^{2}$ Zhejiang Hongxin Garden Company, Wenzhou, Zhejiang, P.R. China \\ Corresponding author: X.F. Wang \\ E-mail:wxf8080@126.com
}

Genet. Mol. Res. 10 (2): 849-859 (2011)

Received December 13, 2010

Accepted February 25, 2011

Published May 10, 2011

DOI 10.4238/vol10-2gmr1207

\begin{abstract}
Camellia is an economically important ornamental plant that has many uses, such as in beverages, foods and medicines. We examined 15 Camellia cultivars in Wenzhou, China, using RAPD markers and measurements of three traits (petal color, flower diameter, blooming period). PCR amplification with 15 random primers produced 1935 bands, observed at 88 amplification loci; $77 \%$ of the amplified loci were polymorphic, with a mean of 4.5 polymorphic loci per primer. The similarity coefficient ranged from 0.5419 to 0.7933 among the 15 samples; the lowest value was between Manao ( $C$. reticulata) and Feibai FR $(C$. japonica), and the largest value was between Chidan $(C$. japonica) and Yuanyang FG ( $C$. japonica). Cluster analysis divided the 15 cultivars into two groups at the similarity coefficient of 0.65 . A correlation was found between RAPD markers and petal color in the first group. No correlation was found between RAPD markers and the other traits (flower diameter, blooming period). This study provides information useful for the identification, classification, phylogenesis, and breeding of Camellia cultivars.
\end{abstract}

Key words: Camellia; Genetic diversity; Correlation; Petal color; Morphological trait; RAPD marker 


\section{INTRODUCTION}

Camellia is an economically important ornamental plant throughout the warm temperate zones, which belongs to the genus Camellia in the family Theaceae (Gao et al., 2005). It can grow well under semi-shaded conditions with wet-warm climate, and features an evergreen appearance, diverse flower colors (red, white, yellow, etc.), graceful flower shapes, and a long blooming period (4-6 months, or even all year) (Gao et al., 2005). Notably, it can bloom in late winter, whereas most plants fail to do so during that time. Additionally, it contains rich volatile oils, vitamins, minerals, antioxidants, and polyphenols. Therefore, it is often used as a raw material for tea beverages, edible oils, spices, traditional herbal medicine, and cosmetic candidates (Ferrara et al., 2001; Kim et al., 2001; Gao et al., 2005; Khan and Mukhtar, 2007; Jung et al., 2007).

So far, over 15,000 Camellia cultivars have been identified and documented in horticulture, which mainly belong to $C$. japonica, $C$. reticulata, $C$. sasanqua, and C. nitidissima (Gao et al., 2005), and new Camellia cultivars still gradually occur as a result of frequent hybridization and artificial selection under cultivation or natural surrounding. Notably, almost all Camellia cultivars are named according to morphological traits such as flower shape and color, and tree or leaf shape. Therefore, disagreement on or discrepancy in the classification of Camellia cultivars inevitably occurs occasionally. On the one hand, it is very difficult to distinguish extremely similar morphological traits such as flower color or shape, and different investigators may draw different conclusions on the same cultivar. On the other hand, some cultivars that have a relatively close genetic relationship show a relatively large morphological difference due to growing changes in surroundings. Comparatively, genomic DNA represents the complete genetic information of species (or cultivars) and is not affected by surroundings, climate and developmental phase. Therefore, molecular techniques are more objective and make it possible to identify, classify or characterize genetic relationships between samples (Xiao and Clifford, 2003).

Presently, the characterization of plant genetic diversities or relationships is mainly performed based on polymerase chain reaction (PCR) amplification or sequence analysis approaches, including RFLP (restriction fragment length polymorphism), ISSR (inter-simple sequence repeat), RAPD (random amplified polymorphic DNA), AFLP (amplified fragment length polymorphism), and SNP (single nucleotide polymorphisms) (Powell et al., 1996; Khlestkina and Salina, 2006). Recently, new approaches such as nrITS (nuclear ribosome internal transcribed spacer) (Vijayan et al., 2009) and DNA barcode (such as plastid DNA sequence) (Kress et al., 2005) are popularly applied to analyze genetic diversities and relationships. Generally, each approach features its particular sensitivity and applicability. In comparison, RAPD analysis could cover the whole genome information, and is still a convenient, effective and low-cost technology, which has been widely and successfully applied to explore genetic relationships (Dorokhov and Klocke, 1997; Ahlawat et al., 2010; Leal et al., 2010). Briefly, it could amplify the whole genomic DNA by the PCR technique with a set of 10-nt random primers with each primer having a specific binding site(s) in genomic DNA. Therefore, the information of amplification products by all primers could disclose genetic diversities among samples.

Camellia features rich genetic diversities (Chung and Kang, 1996). Ueno et al. (1999, 2002) investigated the genetic structure and spatial distribution of individuals within a population of $C$. japonica, who disclosed high diversities and no significant differences in allele frequencies among different size-classes. Xiao and Clifford (2003) identified the genus Camellia by analyzing DNA sequences of the RPB-2 gene (nuclear RNA polymerase II), and provided 
strong support for the hypothesis that the genus Camellia is a monophyletic group. However, they obtained conflicting results concerning partial sub-generic divisions and debated sections of the genus Camellia compared with previous studies. Tang et al. (2006) analyzed the genetic diversities and population structure of six natural populations of $C$. nitidissima from Guangxi (China) based on RAPD and AFLP approaches. They believed that six populations could be classified into two major genetic groups, and also disclosed significant correlations between genetic groups and geographic distances among samples; Wei et al. (2008) analyzed the genetic structure of 13 populations of C. nitidissima by ISSR marker; Vijayan et al. (2009) determined the molecular taxonomy of Camellia by analyzing nrITS sequences. They proposed that 112 species were divided into 8 major clades and 4 isolates; Liu and Gu (2009) believed that $C$. reticulata and $C$. japonica were closely related and that $C$. japonica had partly contributed to the origin of the polyploidy of $C$. reticulata, based on GISH (genomic in situ hybridization).

The previous investigations provided useful information for deeply exploring $\mathrm{Ca}$ mellia genetic diversities or relationships. However, challenges still exist due to the lack of enough molecular information to establish a general standard for the classification and identification of Camellia. Therefore, more investigations are still needed to further explore the genetic diversities of Camellia.

\section{MATERIAL AND METHODS}

\section{Plant material and genomic DNA extraction}

A total of 15 Camellia cultivars were collected from Zhejiang Hongxin Garden Company (Wenzhou, China). They were representative of the major cultivars in Wenzhou, China (Table 1). Genomic DNA was extracted from tender leaf tissue with a modified CTAB method (Porebski et al., 1997). The purity and quantity of genomic DNA were determined by spectrophotometry (Biomate 5, Thermo Electron Corp.) and 1.5\% agarose gel electrophoresis (Amersham Ecl, Electrophoresis power supply-Eps.301), respectively.

Table 1. Samples' name, serial number and morphological traits.

\begin{tabular}{|c|c|c|c|c|}
\hline Code & Name & Petal color & $\mathrm{FD}(\mathrm{cm})$ & $\mathrm{BP}$ \\
\hline N1 & Wuhe PZ (C. reticulata) & Red & $8-10$ & Mar-Apr \\
\hline N2 & Xiuqiuhua (C. japonica) & Red or dark red & $7-9$ & Nov-Mar \\
\hline N3 & Nuo'Er'Si (C. japonica) & Red & $7-9$ & Nov-Mar \\
\hline N4 & Manao (C. reticulata) & Pink & $7-9$ & Dec-Apr \\
\hline N5 & Dafugui (C. japonica) & Pink & $8-11$ & Feb-Mar \\
\hline N6 & Seroyang (C. japonica) & Red with white piece & $8-11$ & Feb-Apr \\
\hline N7 & Chidan (C. japonica $)$ & Red & $10-13$ & Feb-Mar \\
\hline N8 & Yuanyang $\mathrm{FG}(C$. japonica $)$ & Red with white piece & $11-13$ & Feb-Mar \\
\hline N9 & Fenshi YJ (C. japonica) & Pink & $5-6$ & Feb-Mar \\
\hline N10 & Baishi YJ (C. japonica) & White with pink strip & $5-6$ & Feb-Mar \\
\hline N11 & Qixinghong (C. japonica) & Dark red & $7-8$ & Mar-Apr \\
\hline N12 & Huajixiang (C. japonica) & Pink with white piece & $11-13$ & Jan-Mar \\
\hline N13 & Mark Allan (not identified) & Wine red & $11-13$ & Sep-Feb \\
\hline N14 & Feibai FR (C. japonica) & Red & $8-13$ & Jan-Mar \\
\hline N15 & Mary Agnes Patin (not identified) & Rosy red & $11-14$ & Jan-Feb \\
\hline
\end{tabular}

Camellia names according to Gao et al. (2005). FD = flower diameter; $\mathrm{BP}=$ blooming period. 


\section{RAPD-PCR amplification}

Fifteen 10-nt random primers (Table 2) were screened out of 202 primers (S1-S50, S100-S150, S200-300), which were synthesized by the Shanghai Bioengineering Company (Shanghai, China). PCR amplification reaction and program were as described by Wang et al. (2010). All PCR products were separated by $1.2 \%$ agarose gel electrophoresis in $0.5 \mathrm{X}$ TBE buffer (44.5 mM Tris-HCl; $44.5 \mathrm{mM}$ boric acid; $1 \mathrm{mM}$ EDTA). The bands were then visualized by ethidium bromide staining for 15-20 min and photographed under UV light by a Gel Doc-It Imaging system (UVP, Bio Doc Co., USA). The parallel amplification experiments were performed at least two times until distinct and reproducible bands occurred.

Table 2. Primers and their sequences used in this study.
\begin{tabular}{llclcl}
\hline Primer & Sequence & Primer & Sequence & Primer & Sequence \\
\hline S10 & CTGCTGGGAC & S 43 & GTCGCCGTCA & S256 & CTGCGCTGGA \\
S11 & GTAGACCCGT & S 112 & ACGCGCATGT & S 263 & GTCCGGAGTG \\
S20 & GGACCCTTAC & S 128 & GGGATATCGG & S 265 & GGCGGATAAG \\
S32 & TCGGCGATAG & S 216 & GGTGACGCT & S 267 & CTGGACGTCA \\
S39 & CAAACGTCGG & S 254 & TGGGTCCCTC & S 300 & AGCCGTGGAA \\
\hline
\end{tabular}

\section{Data processing and cluster analysis}

All gel bands were scored as present (1) or absent (0) in each genotype for each primer. The band number scoring data were used to calculate the similarity (S) matrixes as described by Nei and Li (1997). A dendrogram was constructed based on similarity matrix using cluster analysis with the unweighted pair group method with arithmetic average (UPGMA).

\section{RESULTS}

\section{Analysis of genotype polymorphism among the Camellia cultivars}

Figure 1 shows the amplification results from partial 10-nt random primers (S20, S112) to 15 genomic DNA samples, and amplification size ranged from 0.3 to $4.0 \mathrm{~kb}$ or so. A total of 1935 bands were observed at 88 amplification loci on 15 electrophoretic gels, and 68 amplification loci were polymorphic, indicating percentage of polymorphic loci of $77.3 \%$ with an average of 4.5 polymorphic loci per primer. A similarity coefficient between 15 samples ranged from 0.5419 to 0.7933 (Table 3), in which the lowest value was 0.5419 between N4 (Manao, C. reticulata) and N14 (Feibai FR, C. japonica), implying the farthest genetic relationship, and the largest value was 0.7933 between N7 (Chidan, $C$. japonica) and N8 (Yuanyang FG, C. japonica), disclosing the closest genetic relationship among the 15 samples. 

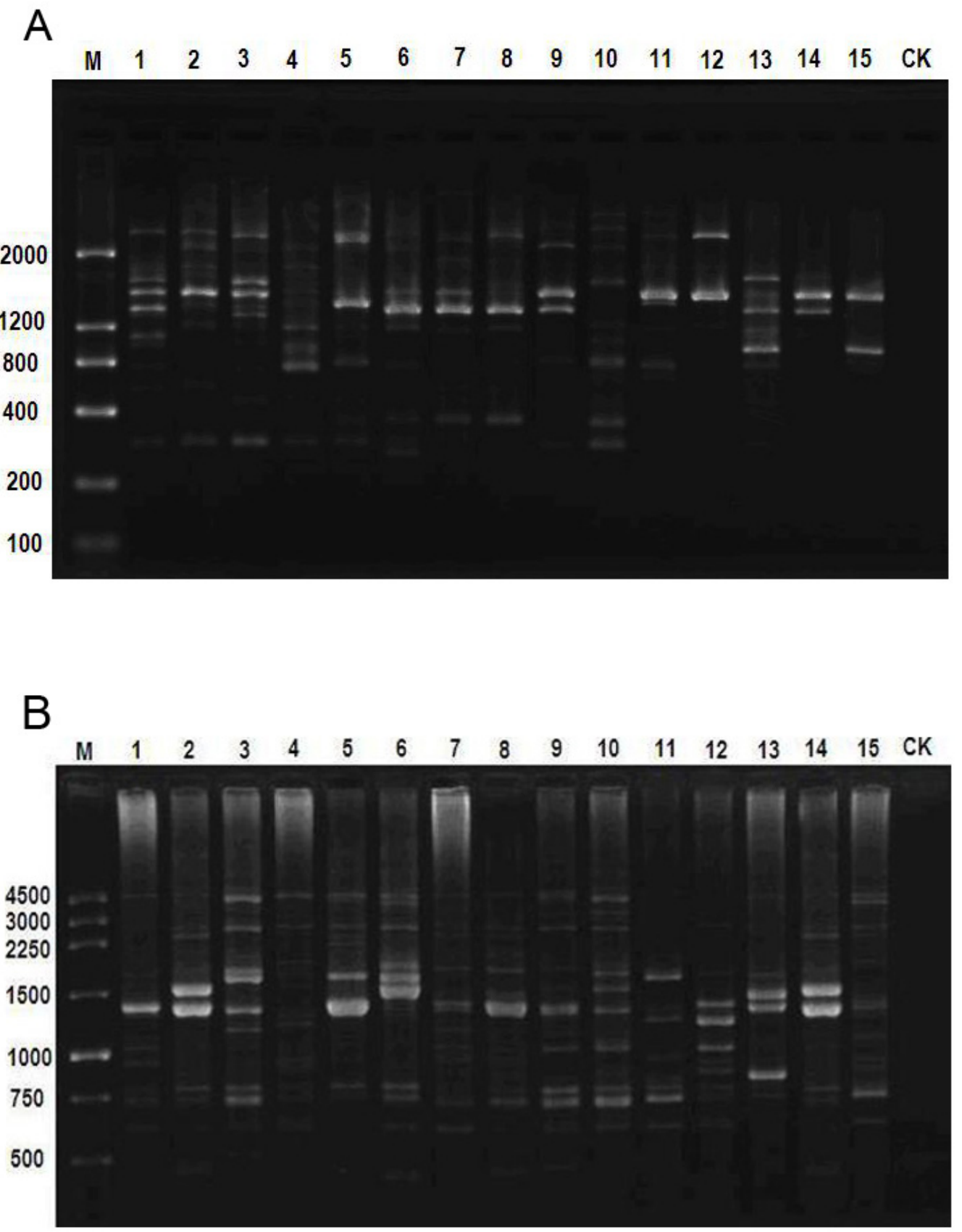

Figure 1. A. Amplification products by polymerase chain reaction using primer S20. B. Amplification products by polymerase chain reaction using primer S112. Lanes 1-15 are identified in Table 1. M = DNA marker; $\mathrm{CK}=$ the negative control. 
X.F. Wang et al.

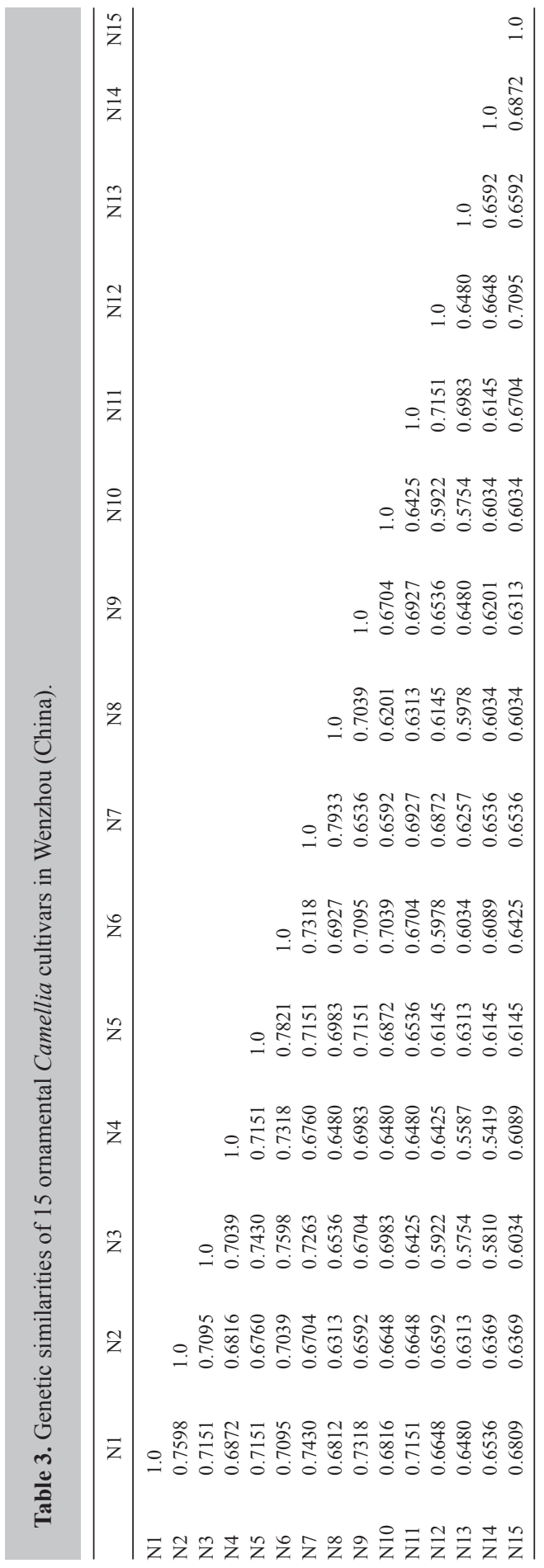




\section{Cluster analysis}

Figure 2 discloses that 15 Camellia cultivars were distinctly clustered into two groups with a similarity coefficient cut-off of 0.65 : N1-N10 in the first group and N11-N15 in the second. In addition, both groups could be divided at a similarity coefficient of 0.68: nine samples (N1 to N9) were in the same cluster while N10 was isolated from the first group, whereas five samples (N11 to N15) were divided into three parts, in which N11, N12, N15 clustered together while N13, N14 were isolated from the second group.

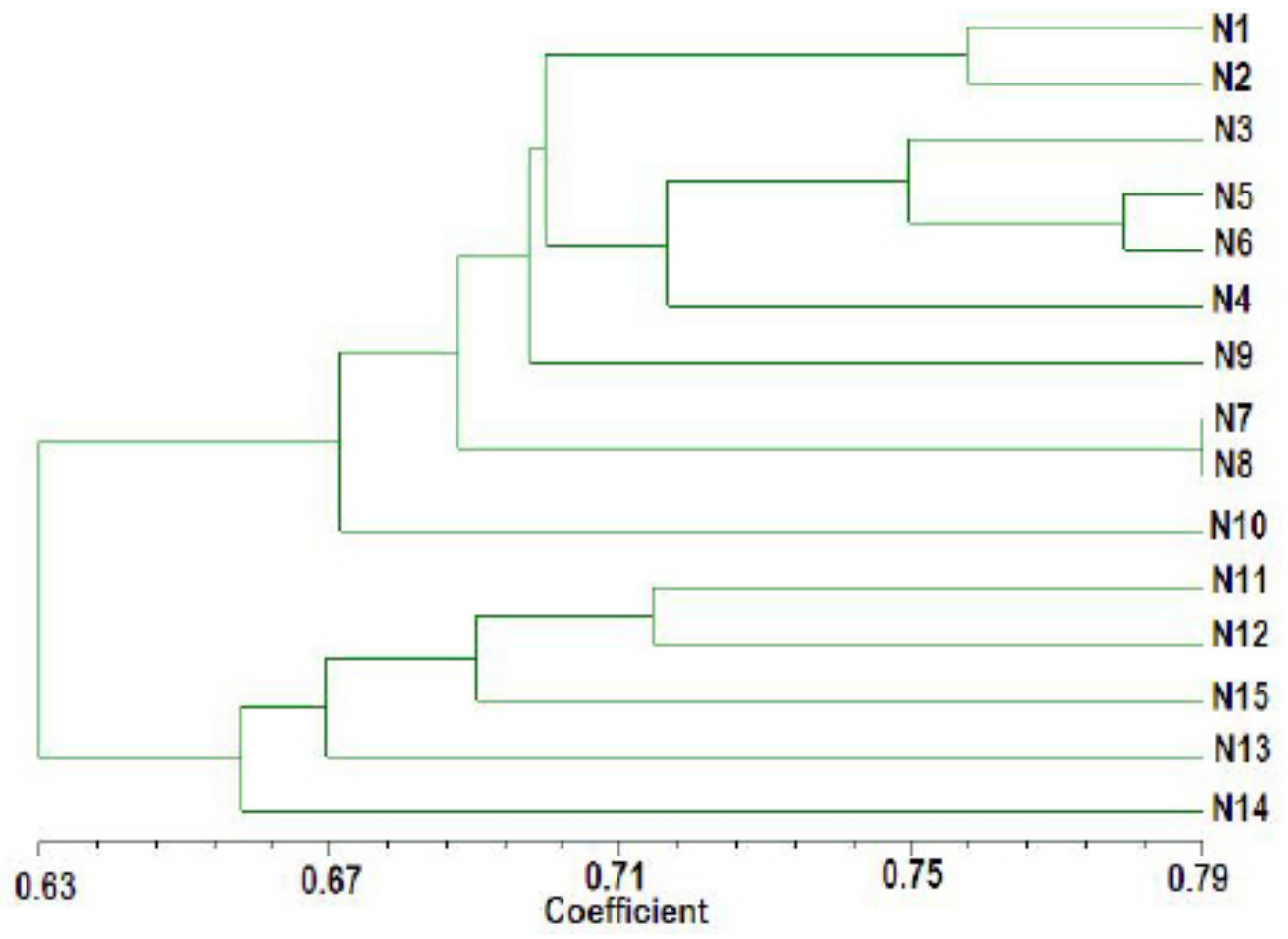

Figure 2. UPGMA dendrogram of 15 samples by RAPD analysis using 15 primers.

\section{Morphological trait analysis}

Table 1 describes three important morphological traits of 15 samples: petal color, flower diameter and blooming period. All samples shared approximate petal color (red or pink) with the exception of sample N10 (white). For flower diameter, two samples (N9 and N10) belonged to small-sized flowers $(5-6 \mathrm{~cm})$, five samples (N7, N8, N12, N13, N15) to large-sized flowers $(10-13 \mathrm{~cm})$, and the remaining 8 samples to middle-sized flowers (7-10 $\mathrm{cm})$. For blooming period, three samples (N2, N3, N4) belonged to early blooming cultivars, and two samples (N1 and N11) to late blooming cultivars, whereas the remaining 10 samples belonged to intermediate blooming cultivars. Figure 3 shows the flower organ and color of some Camellia cultivars. 

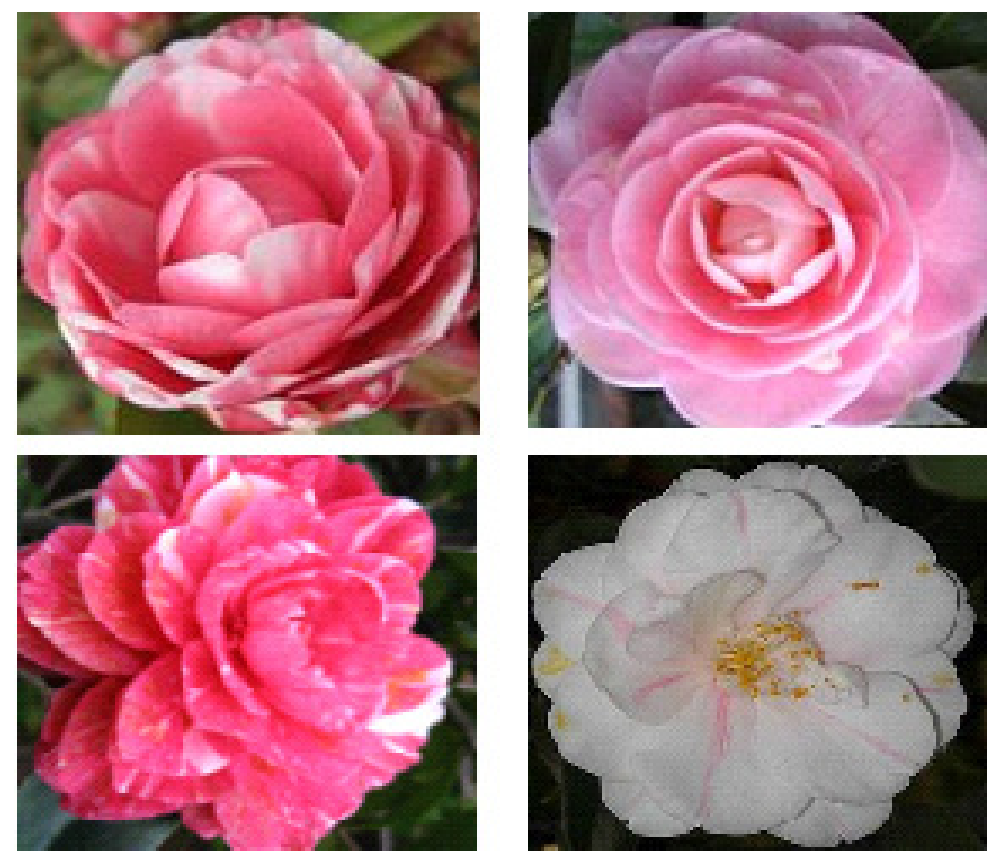

Figure 3. Flower shape and color of partial Camellia cultivars (Upper: N9, Fenshi YJ (C. japonica) and N4, Manao (C. reticulata); Lower: N8, Yuanyang FG (C. japonica) and N10, Baishi YJ (C. japonica)).

\section{DISCUSSION}

Different molecular marker approaches or techniques have their particular sensitivity and applicability. In comparison, the RFLP approach covers a small volume of information, ISSR markers have limited binding sites to genomic DNA, and AFLP markers feature a complicated operation process due to relatively high stability. For SNP analysis, it is costly and labor-intensive, though the approach could disclose relatively true genetic diversities. Recently, new approaches such as nrITS and plastid DNA sequence analysis have been applied to explore genetic diversities, but both approaches also disclose a small volume of local information of the genome. For RAPD markers, there are some doubts because of their relatively low reproduction (or stability). However, this problem of reproduction (or stability) could be easily solved by using high-quality genomic DNA, optimized reaction system and program in a standard operation. In the present study, we acquired clear, stable and reproducible amplification bands with an optimized system and procedure (Wang et al., 2010). Therefore, RAPD markers still provide a rapid and useful technique to investigate genetic diversities at the whole genome level (Ahlawat et al., 2010; Ahmad et al., 2010; Leal et al., 2010).

Camellia features rich genetic resources through long-time hybridization and artificial selection (Chung and Kang, 1996). The present investigation disclosed that all 15 random primers had rich binding sites (1935 bands at 88 amplification loci) in 15 samples of genomic DNA, and also uncovered $77.3 \%$ polymorphic loci. There were large differences in the similarity coefficient ( 0.5419 to 0.7933 ), which implied extensive genetic variation among 15 Camellia cultivars. 
In horticulture, the most important morphological trait could be the floral organ for flowering plant, including floral structure (sepals, petals, stamens, and carpels), flower color and blooming period. These traits are strictly spatially and temporally governed and regulated by a series of genes during the course of different developmental phases, which result in the synthesis of flavonoids and carotenoids, $\mathrm{pH}$ change of enchylema, development of floral organs, and finally formation of floral structures (Vandenbussche et al., 2003; Sablowski, 2010). Here, three morphological traits, including petal color, flower diameter and blooming period, were selected for study. In the first group, morphological analysis disclosed that nine samples (N1 to N9) featured similar petal colors (red), while sample N10 did white petal (Table 1). Cluster analysis showed that 9 samples (N1 to N9) clustered together, while sample N10 was isolated at a similarity coefficient of 0.68 (Figure 2). It seemed that there was a correlation between cluster analysis based on molecular markers and morphological analysis based on flower color in the first group. Additionally, samples N9 and N10, were derived from the same Camellia cultivar - Shiyangjing (Chinese traditional cultivar) and share highly morphological similarities except flower organ, particularly petal color (Table 1; Figure 3). However, they represented relatively low genetic similarity $(0.6704)$ (Table 3 ), and were divided into different clusters at a similarity coefficient of 0.68 (Figure 2), which implied that gene(s), governing or regulating metabolic pathways of petal color, could play an important role in RAPD analysis (Wang et al., 2010). For sample N8 (Yuanyang FG), a bud variant of sample N7 (Chidan), both shared extremely high morphological similarities in petal color, flower diameter size and shape, and blooming period (Table 1). Theoretically, compared with the other 13 samples, N7 and N8 should share the highest genetic similarity and cluster most closely, and information data (the highest similarity coefficient of 0.7933 between N7 and N8) and cluster results did confirm this presumption (Table 3; Figure 2). Therefore, we believe that RAPD analysis was sensitive enough to distinguish genetic diversities among Camellia cultivars.

In the second group, five samples featured similar petal color (red) (Table 1). However, distinct differences were observed when considering flower diameter or blooming period. For $\mathrm{N} 14$, its flower diameter ranged from 8 to $13 \mathrm{~cm}$ (from medium to large flower), while that of N11 only from 6 to $8 \mathrm{~cm}$ (small flower) and those of N12, N13, N15 were similar $(11-13 \mathrm{~cm}$; large flower). As for blooming period, five samples also showed distinct differences: N11 (late blooming: March-April), N13 (early blooming: September-February of the following year) and N12/N14/N15 (intermediate time blooming: January-March). Obviously, parallel correlations failed to occur between morphological analysis (flower diameter or blooming period) and cluster analysis based on molecular markers (Figure 2). The failure to detect correlations possibly resulted from the following two phenomena: 1) Most traits are often governed and regulated by a number of genes, whereas each gene possibly governs and regulates more than one trait; 2) Binding sites of each primer often cover the whole genome not only corresponding gene sections, so the whole genome DNA (including all genes) could contribute to RAPD results. That is, all genes governing flower diameter, blooming period, leaf type, growth potential, tree shape, for example, play different roles in RAPD analysis, in which some genes could play dominant roles and others small roles in RAPD analysis. Therefore, extremely complex amplification information interferes with distinctly parallel relationships between genes and traits.

In the past (and even in the present), plant breeders often select hybridization targets with large differences in morphological traits, scarcely considering their genetic background. Therefore, in practice, breeders often have to spend much time in trying luck to get ideal 
trait(s) due to the fact that some hybridization combinations feature distinct morphological differences but they possibly share high genetic similarity in their genomes. Therefore, breeders have few chances to obtain ideal traits when performing hybridization. Therefore, it is necessary and urgent for plant breeders to explore plant genetic diversities at the genome level. Luckily, molecular approaches, such as genetic-modified technique or molecular marker-assisted breeding, have been successfully applied in practice (Varshney et al., 2005; Collard and Mackill, 2008). In this study, based on a comprehensive analysis of morphological traits (petal color, flower diameter, blooming period) and genetic diversities, we found that the combination N10/N13 was the most suitable hybridization targets, and the combination $\mathrm{N} 4 / \mathrm{N} 14$ was the second.

\section{ACKNOWLEDGMENTS}

Research supported by the Department of Education of Zhejiang province, China (Project \#Y200803397), Science and Technology Bureau of Wenzhou, Zhejiang, China (Project \#N2005A012). The authors wish to thank Hongxin Lin, Dahe Zhang and Xizhi Chen for kindly providing materials.

\section{REFERENCES}

Ahlawat A, Katoch M, Ram G and Ahuja A (2010). Genetic diversity in Acorus calamus L. as revealed by RAPD markers and its relationship with $\beta$-asarone content and ploidy level. Sci. Hortic. 124: 294-297.

Ahmad F, Khan AI, Awan FS, Sadia B, et al. (2010). Genetic diversity of chickpea (Cicer arietinum L.) germplasm in Pakistan as revealed by RAPD analysis. Genet. Mol. Res. 9: 1414-1420.

Chung MG and Kang SS (1996). Genetic variation within and among populations of Camellia japonica (Theaceae) in Korea. Can. J. For. Res. 26: 537-542.

Collard BC and Mackill DJ (2008). Marker-assisted selection: an approach for precision plant breeding in the twenty-first century. Philos. Trans. R. Soc. Lond. B. Biol. Sci. 363: 557-572.

Dorokhov DB and Klocke E (1997). A rapid and economic technique for RAPD analysis of plant genomes. Russ. J. Genet. 33: 443-450.

Ferrara L, Montesano D and Senatore A (2001). The distribution of minerals and flavonoids in the tea plant (Camellia sinensis). Farmaco 56: 397-401.

Gao JY, Clifford RP and Du YQ (2005). Collected Species of the Genus Camellia, an Illustrated Outline. Zhejiang Science and Technology Publishing House, Hangzhou.

Jung E, Lee J, Baek J, Jung K, et al. (2007). Effect of Camellia japonica oil on human type I procollagen production and skin barrier function. J. Ethnopharmacol. 112: 127-131.

Khan N and Mukhtar H (2007). Tea polyphenols for health promotion. Life Sci. 81: 519-533.

Khlestkina EK and Salina EA (2006). SNP markers: methods of analysis, ways of development, and comparison on an example of common wheat. Genetika 42: 725-736.

Kim KY, Davidson PM and Chung HJ (2001). Antibacterial activity in extracts of Camellia japonica L. petals and its application to a model food system. J. Food Prot. 64: 1255-1260.

Kress WJ, Wurdack KJ, Zimmer EA, Weigt LA, et al. (2005). Use of DNA barcodes to identify flowering plants. Proc. Natl. Acad. Sci. U. S. A. 102: 8369-8374.

Leal AA, Mangolin CA, do Amaral ATJ, Goncalves LS, et al. (2010). Efficiency of RAPD versus SSR markers for determining genetic diversity among popcorn lines. Genet. Mol. Res. 9: 9-18.

Liu LQ and Gu (2009). Chromosome relationship between Camellia japonica and Camellia reticulate revealed by genomic in situ hybridization. Chromosome Bot. 4: 1-4.

Nei M and Li WH (1997). Mathematical model for studying genetic variation in terms of restriction endonucleases. Proc. Natl. Acad. Sci. U. S. A. 76: 5269-5273.

Porebski S, Bailey LG and Baum BR (1997). Modification of a CTAB DNA extraction protocol for plants containing high polysaccharide and polyphenol components. Plant Mol. Biol. Rep. 15: 8-15. 
Powell W, Morgante M, Andre C and Hanafey M (1996). The comparison of RFLP, RAPD, AFLP and SSR (microsatellite) markers for germplasm analysis. Mol. Breed. 2: 225-238.

Sablowski R (2010). Genes and functions controlled by floral organ identity genes. Semin. Cell Dev. Biol. 21: 94-99.

Tang S, Bin X, Wang L and Zhong Y (2006). Genetic diversity and population structure of yellow Camellia (Camellia nitidissima) in China as revealed by RAPD and AFLP markers. Biochem. Genet. 44: 449-461.

Ueno S, Yoshimaru H, Tomaru N and Yamamoto S (1999). Development and characterization of microsatellite markers in Camellia japonica L. Mol. Ecol. 8: 335-336.

Ueno S, Tomaru N, Yoshimaru H, Manabe T, et al. (2002). Size-class differences in genetic structure and individual distribution of Camellia japonica L. in a Japanese old-growth evergreen forest. Heredity 89: 120-126.

Vandenbussche M, Zethof J, Souer E, Koes R, et al. (2003). Toward the analysis of the petunia MADS box gene family by reverse and forward transposon insertion mutagenesis approaches: B, C, and D floral organ identity functions require SEPALLATA-like MADS box genes in petunia. Plant Cell 15: 2680-2693.

Varshney RK, Graner A and Sorrells ME (2005). Genomics-assisted breeding for crop improvement. Trends Plant Sci. 10: 621-630.

Vijayan K, Zhang WJ and Tsou CH (2009). Molecular taxonomy of Camellia (Theaceae) inferred from nrITS sequences. Am. J. Bot. 96: 1348-1360.

Wang XF, Zheng WH, Zheng HX and Xie QQ (2010). Optimization of RAPD-PCR reaction system for genetic relationships analysis of 15 Camellia cultivars. Afr. J. Biotechnol. 9: 798-804.

Wei X, Cao HL, Jiang YS, Ye WH, et al. (2008). Population genetic structure of Camellia nitidissima (Theaceae) and conservation implications. Bot. Stud. 49: 147-153.

Xiao TJ and Clifford RP (2003). Molecular analysis of the genus Camellia. Int. Camellia J. 35: 57-65. 